\title{
Reputation and Socio-Ecology in Humans
}

\author{
A. Romano ${ }^{1}$, F. Giardini ${ }^{2}$, S. Columbus ${ }^{3}$, E. W. de Kwaadsteniet ${ }^{1}$, D. \\ Kisfalusi $^{4}$, Z. Triki ${ }^{5,6}$, C. Snijders ${ }^{7}$, K. Hagel $^{8}$ \\ ${ }^{1}$ Social, Economic and Organizational Psychology, Leiden University, 2300 RA Leiden, The \\ Netherlands \\ ${ }^{2}$ Department of Sociology, University of Groningen \& Interuniversity Center for Social Science Theory \\ and Methodology (ICS) \\ ${ }^{3}$ Department of Psychology, University of Copenhagen \\ ${ }^{4}$ Centre for Social Sciences - HAS Centre of Excellence, Budapest, Hungary \\ ${ }^{5}$ University of Neuchâtel, Neuchâtel, Switzerland \\ ${ }^{6}$ Stockholm University, Stockholm, Sweden \\ ${ }^{7}$ Human-Technology Interaction group, Eindhoven University of Technology, the Netherlands \\ ${ }^{8}$ Max Planck Institute for Evolutionary Anthropology, Germany
}

\section{Forthcoming in: Philosophical Transactions B}

Keywords: Reputation, Socio-ecology, Culture, Institutions, Gossip

\section{Acknowledgments}

A.R. was supported by the European Research Council (ERC) under the European Union's Horizon 2020 research and innovation programme (AdG agreement ${ }^{\circ}$ 785635; PI Carsten K.W. De Dreu). D.K. gratefully acknowledges the funding from the European Research Council (ERC) under the European Union's Horizon 2020 research and innovation program (grant agreement No 648693; PI: Károly Takács). Z.T. was supported by the Swiss Science National Foundation (grant number: P2NEP3_188240). 


\section{Reputation and Socio-Ecology in Humans}

\section{Summary}

Reputation is a fundamental feature of human sociality as it sustains cooperative relations among unrelated individuals. Research from various disciplines provides insights on how individuals form impressions of others, condition their behaviors based on the reputation of their interacting partners, and spread or learn such reputations. However, past research has often neglected the socio-ecological conditions that can shape reputation systems and their effect on cooperation. Here, we outline how social environments, cultural values, and institutions come to play a crucial role in how people navigate reputation systems. Moreover, we illustrate how these socio-ecological dimensions affect the interdependence underlying social interactions (e.g., potential recipients of reputational benefits, degree of dependence) and the extent to which reputation systems promote cooperation. To do so, we review the interdisciplinary literature that illustrates how reputation systems are shaped by the variation of prominent ecological features. Finally, we discuss the implications of a socioecological approach to the study of reputation and outline potential avenues for future research.

\section{Introduction}

Reputation is a pervasive feature of human social interactions [1] and can be broadly defined as the set of beliefs, perceptions, and evaluations that an individual has about another person [2]. Reputation serves several functions that sustain beneficial relations within and beyond groups, and it is considered a "universal currency" of social interactions [1]. Reputation helps establish cooperative interactions through successful deterrence of free-riders [2-5] and can sustain cooperation without repeated interactions [2-4]. Two mechanisms by which reputation systems enable cooperation are indirect reciprocity and partner choice [3,6-8]. In indirect reciprocity, an individual A cooperates or defects against B; a third party $\mathrm{C}$ then conditions her decision whether to cooperate with A on A's prior behavior towards B. In partner choice, C uses the information about A's prior behavior to decide whether to choose $\mathrm{A}$ as an interaction partner. Consequently, there are good reasons why people care about the impression they make on others [9]. Such impressions are not only driven by direct observation of one's behavior, but also indirectly by information spread (e.g., gossip) $[1,2]$.

Past research has focused on the factors that play a crucial role in facilitating cooperation with strangers $[2,10-14]$. The two factors of reputation systems that promote cooperation are observability and gossip. When interactions are observable by others, people can become concerned about their reputation among these third parties. Consequently, people are more cooperative in public, than private situations [15-18]. Observability can even sustain cooperation in otherwise anonymous peer-to-peer markets [17]. Reputations are also formed because of what people learn from others about potential collaborators, i.e., gossip. Gossip, compared to other factors such as costly punishment, can be an efficient and low cost way to promote cooperation in groups [2,1922]. Gossip can spread reputational information beyond direct observers, thus providing information about potential partners before actually meeting them [23-25]. Past research shows that gossip is prevalent in traditional small-scale societies [26-28] but also in contemporary large-scale societies [21]. Lab experiments on gossip have provided controlled evidence that individuals are more cooperative when facing the "threat of gossip" [29-31], and modelling shows that gossip supports the evolution of cooperation, even when it is not completely accurate [32]. Although this research provides evidence for the importance of gossip and observability in promoting cooperation, it is less clear the extent to which reputation systems are shaped by socio-ecological conditions. Moreover, there is little research on how interdependence among individuals specifies the consequences of reputation systems for cooperative behavior.

Here we define reputation systems as the set of factors that affect the formation, spread, and use of reputations (such as gossip and observability). Reputation systems do not occur in a vacuum, but are shaped by a rich set of situational and relational factors and the interplay between the individuals and their environments, which we will term more broadly as socio-ecological conditions. Little is known about the socio-ecological factors that might shape and facilitate the different ways in which reputations are formed, spread, and used. Empirical studies have mainly focused on how reputation and opportunities for forming or spreading reputations affect social behavior in a given context, but much less attention has been devoted to different environments, cultural values, and institutions in which different reputation systems emerge and are used. This is in contrast with recent theoretical and empirical efforts that have highlighted the importance of social ecology in shaping 
social behaviors within and across societies [33-35]. Similarly, social-ecological conditions may give rise to reputation systems and shape their particular form.

Additionally, social-ecological conditions may shape the pattern of interdependence that characterizes people's interactions, such as the degree to which they need to rely on others or on themselves (i.e., degree of dependence) or face corresponding vs. conflicting interests [36-40]. They may also shape whether interactions are within the close network or extend to new interaction partners, including strangers and outgroup members $[9,16,44,59]$. Evolutionary models of reputation-based cooperation, such as models of indirect reciprocity [3], have largely overlooked such factors. With a few exceptions [42-45], these models ignore variation in the environments and social contexts in which interactions occur. This results in the often-implicit assumption that the insights from such models are universal, i.e., that they apply and are suitable across diverse institutional, cultural, and environmental conditions.

We propose that variation across social ecologies (a) gives rise to different reputation systems and (b) shapes the interdependence among individuals, which in turn affects the functioning of reputation systems (see Figure 1). Below, we describe a selection of relevant socio-ecological dimensions, such as social conditions, subsistence environments, and cultural and institutional settings, that may influence the efficiency of reputation systems in humans. In taking stock from evidence across different disciplines, we present an overview on the impact of different socio-ecological conditions in shaping reputation systems and interdependence, and we relate them to various consequences for cooperation in humans. Finally, we outline potential avenues on how future research may incorporate a socio-ecological approach into the study of reputation in humans. It is important to note that the list of socio-ecological conditions highlighted in the present review is not exhaustive. Instead, we focus on selected examples that highlight the benefits of considering the socio-ecological context in the study of reputation systems and cooperation.

\section{A socio-ecological approach to reputation}

\section{(a) Social Environment}

The social environment may shape reputation systems. Here, we focus on factors that can affect the information flow and the likelihood of repeated interactions. Two such social environmental factors are network density and population density. In societies characterized by greater population density, the greater frequency of repeated interactions facilitates the extent to which social interactions are observable by others. Moreover, the higher flow of information facilitates how people spread and use reputation information through gossip. Given better opportunities to observe and gossip, we may expect that in higher density areas people will cooperate and trust more compared to areas with lower population density. In a survey involving 5000 business leaders across 31 regions in China, researchers found that business people tend to consider interactions with people from regions with a higher degree of population density as more cooperative, than regions with a lower population density [46]. Similarly, theoretical and experimental studies that manipulate the density of social networks (i.e., the number of connections among individuals within a network) found that denser networks facilitate the spread of information and increase the degree to which certain actions can be observed by others $[47,48]$. This in turn makes reputation systems (e.g., via gossip) more successful in promoting cooperation in denser networks, compared to less dense networks. For example, a recent study conducted among 364 US mTurkers found that denser networks dynamically emerged with cooperation when participants were provided the possibility to observe each other's action [49]. A current day example is the availability of online markets, which can be conceived as creating a dense network of business partners that can deter opportunistic and reward friendly behavior $[50,51]$.

Societies also vary in the extent to which individuals develop interpersonal relationships based on their individual preferences [34,52]. Individuals living in contexts that favor higher relational mobility are free to interact with strangers and to form new relationships (as well as leave old ones). In contrast, in contexts characterized by lower relational mobility interactions are bounded within families and close networks [34]. Therefore, most social interactions are observed by ingroup members, and ingroup members are more often targets of gossip. These differences in mobility can thus have dramatic effects on reputation systems and the way interactions with strangers are framed. In highly relationally mobile societies, people need to possess skills to understand the reputation of potential strangers, and strategically condition their behavior to assure cooperative and beneficial future alliances when observed by others. As a result, people in more mobile societies may rely more on their own and others' reputations in interactions with strangers and be more attentive when gossip and opportunities for social visibility are present. In line with this hypothesis, past research shows 
that Facebook users $(n=116)$ in the highly mobile US encounter more gossip on social media than Facebook users in the less mobile Japan [53]. In contrast, people in less relationally mobile societies may be more concerned about their reputation among ingroup members, leading them to behave with greater conformity when observed and to disclose less about themselves to close others [54,55]. Recent cross-cultural research, involving more than 18,000 participants from industrialized societies, found that people living in societies with higher relational mobility cooperate more with strangers, compared to those living in societies with lower relational mobility [13]. Further research may explain whether such cross-cultural differences in cooperation could occur because people in highly relationally mobile environments rely more on reputation systems (e.g., gossip and observability). Another interesting avenue for future research would be to compare the prevalence of gossip and observability across social environments and to look for invariant traits, such as features of reputation systems that do not depend on variation in the social environment.

\section{(b) Subsistence Environment}

Another prominent environmental factor is the historically prevalent subsistence type within a particular society, defined by its primary food production system [56]. Such more distal features of the ecology (e.g., defensibility and presence of resources) may have influenced the contemporary social ecology [57-59]. In particular, subsistence type might play a role in shaping features of interdependence, that can moderate the effect of reputation systems on cooperation. In particular, societies characterized by different subsistence types differ across relevant dimensions such as dependence among individuals, the prevalence of correspondence vs conflict of interests situations, and/or whether interactions are prevalent within the close network or extend to new partners, like strangers and outgroup members [60,61]. These changes in the degree of interdependence among members of a population may affect the extent to which reputation systems promote cooperation. In fact, it is important to select cooperative partners when individuals have a conflict of interest (i.e., one of the partners needs to sacrifice), or when they depend on other individuals [62]. In such situations, people display higher cooperation when observed by others [62]. For example, herders move residence more often to manage their herd. This often leads them to be involved in situations of conflict of interests, with high dependence on others [56]. Therefore, herders can be sensitive to observability and potential gossip when interacting with strangers. In contrast, farmers lead a more sedentary life and are used to interact with and being observed by ingroup members [56]. A recent large-scale survey, involving residents of 408 Japanese communities $(n=7,295)$, found that people belonging to farming communities are more concerned about their reputation with fellow members, compared to people in non-farming communities [36]. These findings support the idea that these differences in social interactions (affected by the subsistence type) might moderate the relation between reputation systems and cooperation. In a follow-up survey ( $n=1,714,86$ communities), farming communities with substantial reputational concern, a psychological mechanism that is enhanced by observability and gossip [30], have been observed to increase ingroup cooperation up to two years later [36]. Thus, subsistence type may shape whether reputation systems are closely group-bounded or more open, and have consequences for cooperation [44].

There can also be important differences between societies engaged in similar modes of subsistence. For example, rice farming requires farmers to be dependent on each other to build and share irrigation systems with close neighbors, and this leads to highly reciprocal and cooperative relations among neighbors [63]. Wheat farmers, however, do not face the same environmental and social challenges to grow wheat and could therefore restrict their interactions to a smaller circle [60]. It is thus plausible to hypothesize that such differences in cooperation are driven by how, across subsistence types, people react to gossip and observability in interactions with ingroup members or strangers. Further research on the link between subsistence environments and reputation systems might provide interesting insights that would enrich our understanding of different cooperation strategies across contexts.

\section{(c) Cultural values}

Cultural values can be defined as trans-situational goals and principles that are considered important in a given society and guide people's actions [64,65]. Some cultural values that are predominant in a specific society can be associated with variation in interdependence, that in turn may moderate the effect of reputation systems on cooperation among strangers. For example, societies may differ in the degree to which individuals conceptualize the self and prioritize individual vs group goals [66]. Societies characterized by higher collectivistic values are associated with greater levels of interdependence with ingroup members [66]. Researchers have argued that in contexts characterized by higher collectivistic values, people often engage in gossip toward ingroup members to blame deviant behavior, as people care about their reputation primarily in interaction with ingroup members [67]. In line with this, previous research involving employees across 10 
organizations in Pakistan $(n=198)$, reported that a collectivistic orientation moderated the effect of negative gossip on job performance (a proxy of cooperation in the workplace, [68]). On the other hand, societies characterized by a higher prevalence of individualistic values are associated with greater independence from strangers and autonomy, loose connections to ingroups and prioritize personal needs and goals. Although there is no direct evidence for such pattern, a previous cross-cultural study conducted across 16 societies $(n=1,120$, student samples) found that in contexts characterized by a higher degree of individualism people were more cooperative toward strangers, than in more collectivistic contexts [69]. It is possible that such effect on cooperation is mediated by reputation systems and moderated by differences in interdependence across individualistic and collectivistic contexts (Figure 1).

Cultural values may also directly affect reputation systems, and in turn cooperation. For example, societies can also vary based on whether they are characterized by relatively tighter or looser norms [70]. Tighter cultural values are defined by stronger norms, lower tolerance of deviant behavior, and are more likely to have autocratic governments that suppress dissent and media institutions with restricted content. Looser cultural values are characterized by weaker social norms and higher tolerance of deviant behaviors. In contexts characterized by tighter cultural values, institutions play a major role in enforcing cooperation and punishing deviant behaviors, lessening the need to spread information informally [70,71]. Then again, in contexts with tighter norms, norm violations are considered more important so that, all else equal, the necessity of reputation systems in detecting norm violators increases compared to societies with relatively more prevalent looser norms. Although the evidence on the link between tight vs loose societies, reputation, and cooperation is scarce, a recent game theoretic agent-based model found that tighter contexts adopt a cooperative norm faster than looser contexts [72]. An empirical investigation of the cross-cultural variation among different cultural values (such as tightness vs looseness) that affect different reputation systems (and their effect on cooperation) would provide an interesting avenue for future research, also allowing for interdisciplinary collaborations between anthropology and psychology.

\section{(d) Institutions}

Institutions are "the rules of the game" that regulate social interactions in a given society [73-75]. They define what is possible and desirable by incentivizing specific behaviors and prohibiting others. Some institutions are informal, based on the shared understanding of members of a population [73]. Formal and informal institutions can affect the payoff of unwanted behavior. Institutions produce, enact, and enforce norms, which mitigate uncertainty and threat $[76,77]$. They make possible the creation of shared expectations about how people will behave across situations, which facilitates coordination and cooperation [78].

Institutions can also shape how reputations are formed, spread, and used. For example, groups and communities may have different informal norms about what can, and cannot, be communicated through gossip $[26,79]$. Furthermore, formal rules, manifested in official regulations and organizations [74], may prescribe that some reputation-relevant information is collected and made available and observable by public agencies. Economic historians have emphasized the importance of institutions that facilitate the gathering and spreading of reputational information in pre-modern trade. Institutions that promoted the formation and spreading of reputations enabled individuals within the reputation network to profit from cooperation when legal enforcement was impossible. In the absence of ways and means to enforce contracts, medieval merchants relied on reputation-based institutions to support long-distance trade [80-82]. The Jewish Maghribi traders formed an implicit coalition that facilitated the spread of information about contract breaches. The Maghribi traders excluded those who suffered a bad reputation from trading with other members of the coalition $[81,83]$. In Venice, the state provided both rents in the form of trading privileges and protection and intelligence on misbehavior of Venetian merchants abroad. Traders who wanted to benefit from these rents sought a positive reputation with the state, for fear of exclusion [84]. Trading hubs facilitated the spread of reputations through trade networks into early modernity [82,85-87]. Early corporations such as the English East India Company and the Dutch East India Company similarly relied on strategies and governance mechanisms to build up a positive reputation among investors [82].

Contemporary online platforms are inspired by similar principles, and they are pivotal to the establishment of online transactions among distant strangers characterized by asymmetric information, where buyers has limited information about the goods they are going to buy and no way to enforce the terms of the transaction [88]. Such online platforms are institutions that enhance observability by allowing users to monitor and assess reputations within a given community of users $[51,88]$. Indeed, online markets (providing the possibility to observe a positive or a negative review) support exchange, and individuals with a positive reputation benefit [50], even though competitors can artificially manipulate reviews (the own or the others'), and thus portray an 
inaccurate public image [51]. There is increasing evidence pointing to the "dark side" of institutions and more research is needed to understand the negative consequences of these platforms. For instance, reputation systems can support illicit trade, where other forms of norm enforcement are not available [89], or they have unintended negative consequences. Tracking the performance of public services can be very useful for citizens [90], but the same rankings have been indicated as "technologies of global governance" [91], which can affect power relationships and governance. Future research is needed to explore the limitations of reputation systems in online markets, but also their unintended side-effects (e.g., distorted reputational information).

\section{Discussion and conclusions}

Decades of research on reputation have outlined its key role in regulating social behavior in humans. However, past research has overlooked the socio-ecological conditions that influence the relation between reputation systems and cooperation. Here we presented a review on environmental, cultural, and institutional factors that shape reputation systems and how reputation systems can affect cooperation via changes in the interdependence structure. In particular, we highlighted how such factors (e.g., relational mobility, prevalence of individualistic values, formal institutions) could play a crucial role in influencing either $(i)$ the degree to which one's actions are observable, (ii) how easily reputational information can spread through gossip, and (iii) the interdependence underlying social interactions. In turn, this affects the success of reputation-based mechanisms of indirect reciprocity and partner choice in promoting cooperation.

By focusing on a few prominent socio-ecological factors, our review can provide a summary to guide future research on reputation. However, we should also outline a few limitations. First, we focused on a limited set of factors that may influence the form and functioning of reputation systems in particular contexts. Other socioecological factors may also play a crucial role in affecting reputation systems in other contexts. For example, formal and informal religious institutions can also play a prominent role in the study of reputation [76,92]. For example, it has been hypothesized that religion offers a circumscribed social arena in which reputations can be built, evaluated, rewarded and efficiently punished, through religious practices and rituals [93]. Moreover, as religious practices and symbolic markers are successful when interactions are restricted to close ingroup members, in contexts characterized by higher degree of religiosity, reputation systems may be particularly relevant to promote cooperation toward co-religionists [76]. Second, although we showed how socio-ecological conditions might matter, we did not discuss how the interaction between socio-ecological factors (the environment, cultural values, and institutions) can explain variation in reputation systems, interdependence, and cooperation. Rather, we provided an overview on how each specific component might independently influence the reputation system. Future research is needed to understand the interaction between these socio-ecological factors and/or the underlying overlapping mechanisms that these different socio-ecological differences may trigger.

Finally, we only focused on specific aspects of reputation (e.g., reputation systems and their relation with interdependence). However, variation across social ecologies may also affect other aspects of reputation, such as the specific content of reputation information that may promote cooperation [94]. For example, in some foraging immediate-return social ecologies, gaining a reputation for being generous helps establish cooperation within close groups $[43,95,96]$. In fact, in such contexts, a reputation for pro-sociality generates trust among individuals, who are more willing to select as cooperative partners people with such positive reputation [97]. By contrast, in other contexts, a reputation for strength is more relevant and pervasive to regulate social interactions among individuals $[98,99]$. Future research is needed to understand how socio-ecological variation might be associated to other aspects of reputation.

Although previous research has focused on reputation systems in particular societies and contexts [29-31], there is still a tendency to assume that reputation systems operate in a social vacuum, or that they will invariantly be effective in promoting cooperation across diverse contexts. Here, we have illustrated how a variety of socio-ecological conditions might shape the functioning of reputation systems (e.g., through gossip or through observability) and their effects on cooperation. Our review thus aligns with recent calls for incorporating socio-ecology in the study of human social behavior [33-35]. To conclude, the present review provides a conceptual framework and a unified collection of empirical findings to understand reputation systems and their relation to cooperation within and across contexts. 


\section{References}

1. Milinski M. 2016 Reputation, a universal currency for human social interactions. Philos. Trans. R. Soc. B Biol. Sci. 371. (doi:10.1098/rstb.2015.0100)

2. Wu J, Balliet D, Van Lange PAM. 2016 Reputation, Gossip, and Human Cooperation. Soc. Personal. Psychol. Compass 10, 350-364. (doi:10.1111/spc3.12255)

3. Nowak MA, Sigmund K. 2005 Evolution of indirect reciprocity. Nature 34, 183-231. (doi:10.1038/nature04131)

4. Giardini F, Paolucci M, Villatoro D, Conte R. 2014 Punishment and Gossip: Sustaining Cooperation in a Public Goods Game. In Advances in Social Simulation, pp. 107-118. Berlin: Springer Verlag. (doi:10.1007/978-3-642-39829-2_10)

5. Samu F, Számadó S, Takács K. 2020 Scarce and directly beneficial reputations support cooperation. Sci. Rep. 10, 11486. (doi:10.1038/s41598-020-68123-x)

6. Roberts G. 1998 Competitive altruism: From reciprocity to the handicap principle. Proc. R. Soc. B Biol. Sci. 265, 427-431. (doi:10.1098/rspb.1998.0312)

7. Santos FP, Pacheco JM, Santos FC. In press. The complexity of human cooperation under indirect reciprocity. Philos. Trans. R. Soc. B

8. Roberts G, Raihani NJ, Bshary R, Manrique HM, Fariña A, Samu F, Barclay P. In press. The benefits of being seen to help others: indirect reciprocity and reputation-based partner choice. Philos. Trans. R. Soc. $B$

9. Goffman E. 1959 The presentation of self in everyday life. New York: Garden city.

10. Milinski M, Semmann D, Krambeck HJ. 2002 Reputation helps solve the 'tragedy of the commons'. Nature 415, 424-426. (doi:10.1038/415424a)

11. Yamagishi T, Kiyonari T. 2000 The group as the container of generalized reciprocity. Soc. Psychol. Q. 63, 116-132. (doi:10.2307/2695887)

12. Wu J, Balliet D, Peperkoorn LS, Romano A, Van Lange PAM. 2020 Cooperation in Groups of Different Sizes: The Effects of Punishment and Reputation-Based Partner Choice. Front. Psychol. 10, 1-13. (doi:10.3389/fpsyg.2019.02956)

13. Romano, M. Sutter, J. Liu, T. Yamagishi, D. Balliet A. in press. National Parochialism is ubiquitous across 42 nations around the world. Nat. Commun.

14. Romano A, Balliet D, Yamagishi T, Liu JH. 2017 Parochial trust and cooperation across 17 societies. Proc. Natl. Acad. Sci. U. S. A. 114, 12702-12707. (doi:10.1073/pnas.1712921114)

15. Andreoni J, Petrie R. 2004 Public goods experiments without confidentiality: A glimpse into fundraising. J. Public Econ. 88, 1605-1623. (doi:10.1016/S0047-2727(03)00040-9)

16. Filiz-Ozbay E, Ozbay EY. 2014 Effect of an audience in public goods provision. Exp. Econ. 17, 200214. (doi:10.1007/s10683-013-9363-y)

17. Van Vugt M, Hardy CL. 2010 Cooperation for reputation: Wasteful contributions as costly signals in public goods. Gr. Process. Intergr. Relations 13, 101-111. (doi:10.1177/1368430209342258)

18. Ariely D, Bracha A, Meier S. 2009 Doing good or doing well? Image motivation and monetary incentives in behaving prosocially. Am. Econ. Rev. 99, 544-555. (doi:10.1257/aer.99.1.544)

19. Fehr D, Sutter M. 2019 Gossip and the efficiency of interactions. Games Econ. Behav. 113, 448-460. (doi:10.1016/j.geb.2018.10.003)

20. Lindenberg S, Wittek R, Giardini F. 2020 Reputation Effects , Embeddedness, and Granovetter 's Error. Adv. Sociol. Trust Coop. (doi:10.1515/9783110647495-006)

21. Cruz TDD, Nieper A, Testori M, Martinescu E, Beersma B. In press. An Integrative Definition and Framework to Study Gossip. (doi:10.31234/OSF.IO/B8X57)

22. Wu, J., Balliet, D., Van Lange P. 2016 Gossip versus punishment: The efficiency of reputation to promote and maintain cooperation. Sci. Rep. 6, 2319.

23. Ellickson R. 1991 Order without law. Harvard University Press.

24. Barkow JH. 1992 Beneath new culture is old psychology: Gossip and social stratification. . In The adapted mind: Evolutionary psychology and the generation of culture (eds L Barkow, L Cosmides, J Tooby), pp. 627-637.

25. Guala F. 2012 Reciprocity: Weak or Strong? What Punishment Experiments Do (and Do Not) Demonstrate. SSRN Electron. J. (doi:10.2139/ssrn.1640616)

26. Besnier N. 2009 Gossip and the Everyday Production of Politics. University of Hawaii Press.

27. Haviland JB. 1977 Gossip as Competition in Zinacantan. J. Commun. 27, 186-191. (doi:10.1111/j.14602466.1977.tb01816.x)

28. Boehm C. 2012 Gossip and reputation in small-scale societies. In The Oxford Handbook of Gossip and Reputation (eds F Giardini, R Wittek), pp. 254-274.

29. Piazza J, Bering JM. 2007 Concerns about reputation via gossip promote generous allocations in an 
economic game. Elsevier (doi:10.1016/j.evolhumbehav.2007.12.002)

30. Wu J, Balliet D, Van Lange PAM. 2015 When Does Gossip Promote Generosity? Indirect Reciprocity Under the Shadow of the Future. Soc. Psychol. Personal. Sci. 6, 923-930.

(doi:10.1177/1948550615595272)

31. Wu J, Balliet D, PAM Van Lange. 2016 Reputation management: Why and how gossip enhances generosity. Evol. Hum. Behav. 37, 193-201.

32. Giardini F, Vilone D. 2016 Evolution of gossip-based indirect reciprocity on a bipartite network. Sci. Rep. 6, 37931. (doi:10.1038/srep37931)

33. Oishi S. 2014 Socioecological psychology. Annu. Rev. Psychol. 65, 581-609. (doi:10.1146/annurevpsych-030413-152156)

34. Thomson R et al. 2018 Relational mobility predicts social behaviors in 39 countries and is tied to historical farming and threat. Proc. Natl. Acad. Sci. U. S. A. 115, 7521-7526.

(doi:10.1073/pnas.1713191115)

35. Henrich J. 2017 The Secret of Our Success. Princeton University Press.

36. Uchida Y, Takemura K, Fukushima S, Saizen I, Kawamura Y, Hitokoto H, Koizumi N, Yoshikawa S. 2019 Farming cultivates a community-level shared culture through collective activities: Examining contextual effects with multilevel analyses. J. Pers. Soc. Psychol. 116, 1-14.

(doi:10.1037/pspa0000138)

37. Gerpott FH, Balliet D, Columbus S, Molho C, de Vries RE. 2018 How do people think about interdependence? A multidimensional model of subjective outcome interdependence. J. Pers. Soc. Psychol. 115, 716-742. (doi:10.1037/pspp0000166)

38. Aktipis A, Cronk L, Alcock J, Ayers J, ... CB. 2018 Understanding cooperation through fitness interdependence. Nat. Hum. Behav. 2, 429-431.

39. Columbus S, Molho C, Righetti F, Balliet D. 2021 Interdependence and cooperation in daily life. $J$. Pers. Soc. Psychol. 120, 626-650.

40. Tomasello M, Melis AP, Tennie C, Wyman E, Herrmann E. 2012 Two key steps in the evolution of human cooperation: The interdependence Hypothesis. Curr. Anthropol. 53, 673-692. (doi:10.1086/668207)

41. Romano A, Balliet D, Wu J. 2017 Unbounded indirect reciprocity: Is reputation-based cooperation bounded by group membership? J. Exp. Soc. Psychol. 71. (doi:10.1016/j.jesp.2017.02.008)

42. Hawkes K, Bird RB. 2002 Showing Off, Handicap Signaling, and the Evolution of Men's Work. Evol. Anthropol. 11, 58-67. (doi:10.1002/evan.20005)

43. Stibbard-Hawkes DNE. 2019 Costly signaling and the handicap principle in hunter-gatherer research: A critical review. Evol. Anthropol. 28, 144-157. (doi:10.1002/evan.21767)

44. Stibbard-Hawkes DNE, Attenborough RD, Marlowe FW. 2018 A noisy signal: To what extent are Hadza hunting reputations predictive of actual hunting skills? Evol. Hum. Behav. 39, 639-651. (doi:10.1016/j.evolhumbehav.2018.06.005)

45. Righi S, Takács K. 2018 Social Closure and the Evolution of Cooperation via Indirect Reciprocity. Sci. Reports 2018 81 8, 1-9. (doi:10.1038/s41598-018-29290-0)

46. Zhang W, Ke R. 2002 Trust in China: A Cross-Regional Analysis. Econ. Res. J. 10, 59-65. (doi:10.2139/ssrn.577781)

47. Larson JM. 2017 Networks and Interethnic Cooperation. J. Polit. 79, 546-559. (doi:10.1086/690066)

48. Takács K, Gross J, Testori M, Letina S, Kenny A, Power EA, Wittek R. In press. Networks of Reliable Reputations and Cooperation: A Review. Philos. Trans. R. Soc. B

49. Gallo E, Yan C. 2015 The effects of reputational and social knowledge on cooperation. Proc. Natl. Acad. Sci. U. S. A. 112, 3647-3652. (doi:10.1073/pnas.1415883112)

50. Diekmann A, Jann B, Przepiorka W, Wehrli S. 2014 Reputation Formation and the Evolution of Cooperation in Anonymous Online Markets. Am. Sociol. Rev. 79, 65-85.

(doi:10.1177/0003122413512316)

51. Snijders C, Matzat U. 2019 Online reputation systems. In The Oxford Handbook of Gossip and Reputation (eds F Giardini, R Wittek), pp. 479-495. The Oxford Hanbook of Gossip and Reputation.

52. Yuki M, Schug J. 2011 Relational Mobility: A Socio-Ecological Approach to Personal Relationships. In Decade of Behavior 2000-2010. Relationship Science: Integrating Evolutionary, Neuroscience, and Sociocultural Approaches (ed \& AK O. Gillath, G. Adams), pp. 137-151. American Psychological Association. (doi:https://doi.org/10.1037/13489-007)

53. Thomson R. 2016 A Socioecological Approach to Behavior and Psychological Tendencies on Social Network Sites : The Role of Relational Mobility. See https://eprints.lib.hokudai.ac.jp/dspace/handle/2115/61614.

54. Yamagishi T, Hashimoto H, Schug J. 2008 Preferences versus strategies as explanations for culturespecific behavior. Psychol. Sci. 19, 579-584. (doi:10.1111/j.1467-9280.2008.02126.x) 
55. Schug J, Yuki M, Maddux W. 2010 Relational Mobility Explains Between- and Within-culture Differences in Self-disclosure to Close Friends. Psychol. Sci. 21, 1471-1478.

(doi:10.1177/0956797610382786)

56. Nisbett RE, Choi I, Peng K, Norenzayan A. 2001 Culture and systems of thought: Holistic versus analytic cognition. Psychol. Rev. 108, 291-310. (doi:10.1037/0033-295X.108.2.291)

57. Wood BM et al. 2021 Gendered movement ecology and landscape use in Hadza hunter-gatherers. Nat. Hum. Behav. 5, 436-446. (doi:10.1038/s41562-020-01002-7)

58. Kelly R. 2013 The lifeways of hunter-gatherers: the foraging spectrum.

59. Sabloff PLW, Hooper PL, Smith EA, Kohler T, Wright HT, Kaplan HS. 2018 Ecological and Social Dynamics of Territoriality and Hierarchy Formation. Emerg. Premodern States New Perspect. Dev. Complex Soc. , 105-130. (doi:10.37911/9781947864030.05)

60. Talhelm T, Zhang X, Oishi S, Shimin C, Duan D, Lan X, Kitayama S. 2014 Large-scale psychological differences within China explained by rice versus wheat agriculture. Science 344, 603-608.

(doi:10.1126/science.1246850)

61. Witkin HA, Berry JW. 1975 Psychological Differentiation in Cross-Cultural Perspective. J. Cross. Cult. Psychol. 6, 4-87. (doi:10.1177/002202217500600102)

62. Balliet D, Tybur JM, Van Lange PAM. 2016 Functional interdependence theory: An evolutionary account of social situations. Personal. Soc. Psychol. Rev. 21, 361-388. (doi:10.1177/1088868316657965)

63. Talhelm T, Oishi S. 2018 How Rice Farming Shaped Culture in Southern China. Oxford University Press. (doi:10.1093/oso/9780190492908.003.0003)

64. Hitlin S, Piliavin JA. 2004 Values: Reviving a Dormant Concept. Annu. Rev. Sociol. 30, 359-393. (doi:10.1146/annurev.soc.30.012703.110640)

65. Schwartz SH. 1992 Universals in the content and structure of values: Theoretical advances and empirical tests in 20 countries. Adv. Exp. Soc. Psychol. 25, 1-65. (doi:10.1016/S0065-2601(08)60281-6)

66. Triandis H. 2018 Individualism and collectivism. Routledge.

67. Naroll R. 1983 The moral order: an introd. to the human situation. Beverly Hills: SAGE Publications. See https://ixtheo.de/Record/1083866095.

68. De Clercq D, Haq IU, Azeem MU. 2019 Gossiping about outsiders: How time-related work stress among collectivistic employees hinders job performance. J. Manag. Organ. , 1-16.

(doi:10.1017/jmo.2019.56)

69. Herrmann B, Thöni C, Gächter S. 2008 Antisocial punishment across societies. Science 319, 13621367. (doi:10.1126/science.1153808)

70. Gelfand MJ et al. 2011 Differences between tight and loose cultures: A 33-nation study. Science 332, 1100-1104. (doi:10.1126/science.1197754)

71. Harrington JR, Gelfand MJ. 2014 Tightness-looseness across the 50 united states. Proc. Natl. Acad. Sci. 111, 7990-7995. (doi:10.1073/pnas.1317937111)

72. Pan X, Nau D, Gelfand M. 2020 Cooperative Norms and the Growth of Threat: Differences across Tight and Loose Cultures. In Proceedings of 2020 7th IEEE International Conference on Behavioural and Social Computing, BESC 2020, Institute of Electrical and Electronics Engineers Inc. (doi:10.1109/BESC51023.2020.9348297)

73. Guala F. 2016 Understanding Institutions: The Science and Philosophy of Living Together. Princeton University Press.

74. North DC. 1990 Institutions, Institutional Change and Economic Performance. Cambridge University Press. (doi:10.1017/cbo9780511808678)

75. Hodgson GM. 2006 What are institutions? J. Econ. Issues. 40, 1-25. (doi:10.1080/00213624.2006.11506879)

76. Hruschka DJ, Henrich J. 2013 Economic and evolutionary hypotheses for cross-population variation in parochialism. Front. Hum. Neurosci. (doi:10.3389/fnhum.2013.00559)

77. Spadaro G, Gangl K, van Prooijen JW, van Lange PAM, Mosso CO. 2020 Enhancing feelings of security: How institutional trust promotes interpersonal trust. PLoS One 15. (doi:10.1371/journal.pone.0237934)

78. Knight J. 1998 The bases of cooperation: Social norms and the rule of law. J. Institutional Theor. Econ. 154, 754-763.

79. BRENNEIS D. 1984 grog and gossip in Bhatgaon: style and substance in Fiji Indian conversation. Am. Ethnol. 11, 487-506. (doi:10.1525/ae.1984.11.3.02a00050)

80. Greif A. 2006 Institutions and the path to the modern economy: Lessons from medieval trade. Cambridge University Press. (doi:10.1017/CBO9780511791307)

81. Greif A. 1989 Reputation and coalitions in medieval trade: evidence on the Maghribi traders. J. Econ. Hist. 49, 857-882. (doi:https://www.jstor.org/stable/2122741) 
82. Harris R. 2013 Reputation at the Birth of Corporate Governance. Bus. Hist. Rev. 87, 637-640.

83. Greif A. 1993 Contract enforceability and economic institutions in early trade: The Maghribi traders' coalition. Am. Econ. Rev. 83, 525-548.

(doi:https://ezproxy.leidenuniv.nl:2443/login?url=https://www.jstor.org/stable/2117532)

84. Gonzalez de Lara Y. 2008 The secret of Venetian success: a public-order, reputation-based institution. Eur. Rev. Econ. Hist. 12, 247-285. (doi:10.1017/S1361491608002281)

85. Aslanian S. 2014 From the Indian Ocean to the Mediterranean: the global trade networks of Armenian merchants from New Julfa. University of California Press.

86. Margariti RE. 2007 Aden and the Indian Ocean trade: 150 years in the life of a medieval Arabian port. University of North Carolina Press. (doi:10.1017/s1356186307008164)

87. Gelderblom O. 2015 Cities of commerce: the institutional foundations of international trade in the Low Countries, 1250-1650.

88. Dellarocas CN. 2012 Designing Reputation Systems for the Social Web. SSRN Electron. J. (doi:10.2139/ssrn.1624697)

89. Przepiorka W, Norbutas L, Corten R. 2017 Order without Law: Reputation Promotes Cooperation in a Cryptomarket for Illegal Drugs. Eur. Sociol. Rev. 33, 752-764. (doi:10.1093/esr/jcx072)

90. James O. 2011 Performance measures and democracy: Information effects on citizens in field and laboratory experiments. J. Public Adm. Res. Theory 21, 399-418.

91. Davis KE, Kingsbury B, Merry SE. 2012 Indicators as a Technology of Global Governance. Law Soc. Rev. 46, 71-104. (doi:10.1111/j.1540-5893.2012.00473.x)

92. McNamara RA, Norenzayan A, Henrich J. 2016 Supernatural punishment, in-group biases, and material insecurity: experiments and ethnography from Yasawa, Fiji. Relig. Brain Behav. 6, 34-55.

(doi:10.1080/2153599X.2014.921235)

93. Sosis R. 2005 Does Religion Promote Trust?: The Role of Signaling, Reputation, and Punishment. Interdiscip. J. Res. Relig. 1, 1-30.

94. Macfarlan SJ, Lyle HF. 2015 Multiple reputation domains and cooperative behaviour in two Latin American communities. royalsocietypublishing.org 370. (doi:10.1098/rstb.2015.0009)

95. Bliege Bird R, Power EA. 2015 Prosocial signaling and cooperation among Martu hunters. Evol. Hum. Behav. 36, 389-397. (doi:10.1016/j.evolhumbehav.2015.02.003)

96. Bird RB, Smith EA, Bird DW. 2001 The hunting handicap: Costly signaling in human foraging strategies. Behav. Ecol. Sociobiol. 50, 9-19. (doi:10.1007/s002650100338)

97. Power EA, Ready E. 2018 Building Bigness: Reputation, Prominence, and Social Capital in Rural South India. Am. Anthropol. 120, 444-459. (doi:10.1111/aman.13100)

98. Lyle HF, Smith EA. 2014 The reputational and social network benefits of rosociality in an Andean community. Proc. Natl. Acad. Sci. U. S. A. 111, 4820-4825. (doi:10.1073/pnas.1318372111)

99. Cohen D, Bowdle BF, Nisbett RE, Schwarz N, Bowdle BF, Schwarz N. 1996 Insult, aggression, and the southern culture of honor: An 'experimental ethnography.' J. Pers. Soc. Psychol. 70, 945-960. (doi:10.1037//0022-3514.70.5.945) 
Figure 1. Summary of a theoretical framework highlighting the relation between socioecological conditions, reputation systems, and interdependence affecting cooperation toward strangers.

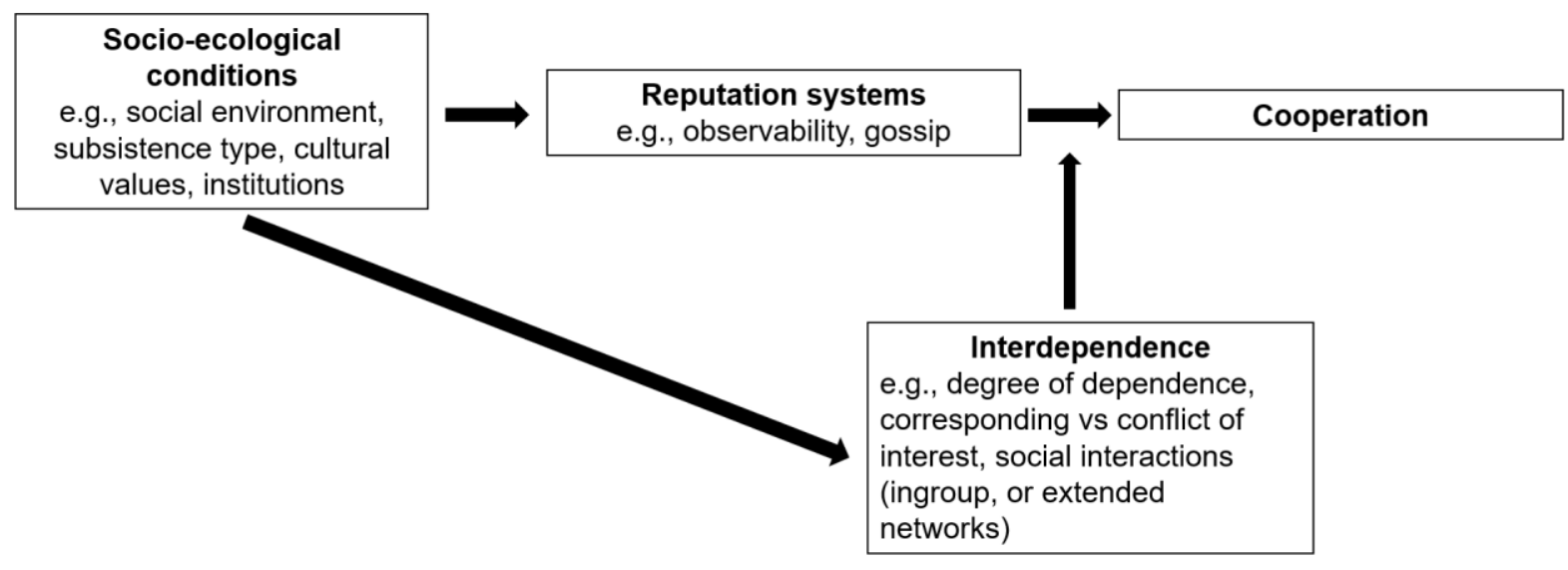

